\title{
Reflets
}

Revue d'intervention sociale et communautaire

\section{Enquête qualitative sur la prise de décision en obstétrique : le choix du mode d'accouchement après césarienne}

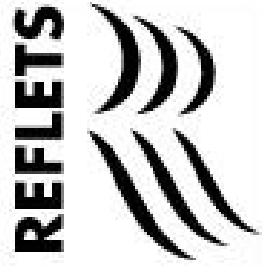

\section{Marylène Dugas et Nils Chaillet}

Volume 17, numéro 2, automne 2011

Représentations, pratiques et expérience dans le champ de santé : tendre des ponts entre des disciplines

URI : https://id.erudit.org/iderudit/1012130ar

DOI : https://doi.org/10.7202/1012130ar

Aller au sommaire du numéro

Éditeur(s)

Reflets, Revue d'intervention sociale et communautaire

ISSN

1203-4576 (imprimé)

1712-8498 (numérique)

Découvrir la revue

Citer cet article

Dugas, M. \& Chaillet, N. (2011). Enquête qualitative sur la prise de décision en obstétrique : le choix du mode d'accouchement après césarienne. Reflets, 17(2), 77-100. https://doi.org/10.7202/1012130ar

\section{Résumé de l'article}

En obstétrique, la médecine offre différentes options pour améliorer les résultats de santé. Ces possibilités représentent autant de prises de décision auxquelles sont confrontés les patients. Afin de procéder au choix des soins, le modèle de partage de la décision médicale constitue actuellement le principal paradigme. Prenant pour exemple le processus de décision concernant le mode d'accouchement après césarienne, nous explorons quelques aspects du processus de prise de décision en obstétrique. De façon générale, la prise de décision concernant le mode d'accouchement après césarienne relève davantage du principe d'autonomie que du partage d'information. Les consultations individuelles ou de groupe, ou encore les outils d'analyse décisionnelle semblent prometteurs pour gérer les différentes problématiques rencontrées.
Tous droits réservés @ Reflets, Revue d’intervention sociale et communautaire, 2012
Ce document est protégé par la loi sur le droit d'auteur. L'utilisation des services d'Érudit (y compris la reproduction) est assujettie à sa politique d'utilisation que vous pouvez consulter en ligne. 


\section{Enquête qualitative sur la prise de décision en obstétrique : le choix du mode d'accouchement après césarienne}

Marylène Dugas, PhD,

CHU Sainte-Justine, Département Obstétrique et Gynécologie, Université de Montréal

Nils Chaillet, $P h D^{1}$

CHU Sainte-Justine, Département Obstétrique et Gynécologie, Université de Montréal

Résumé

En obstétrique, la médecine offre différentes options pour améliorer les résultats de santé. Ces possibilités représentent autant de prises de décision auxquelles sont confrontés les patients. Afin de procéder au choix des soins, le modèle de partage de la décision médicale constitue actuellement le principal paradigme. Prenant pour exemple le processus de décision concernant le mode d'accouchement après césarienne, nous explorons quelques aspects du processus de prise de décision en obstétrique. De façon générale, la prise de décision concernant le mode d'accouchement après césarienne relève davantage du principe d'autonomie que du partage d'information. Les consultations individuelles ou de groupe, ou encore les outils d'analyse décisionnelle semblent prometteurs pour gérer les différentes problématiques rencontrées.

Mots clés : prise de décision partagée, obstétrique, outils d'aide à la décision, éthique 


\section{Abstract}

In obstetrics, several options are offered to improve health outcomes. These possibilities can be considered as many decisions that parents will need to face. To proceed to the choice of care, the model of shared decision making is currently the main paradigm. Taking as an example the decision process regarding the mode of delivery after cesarean section, we explore some aspects of the process of decision making in obstetrics. In general, decision making regarding the mode of delivery after cesarean is more the principle of autonomy then shared decision. Individual or group counseling, or decision analysis tools are promising tools to help with the various problems encountered.

Key words : Shared decision making, Obstetrics, Decision aid Tools, Ethics

\section{Introduction}

Dans le domaine obstétrical, les technologies médicales offrent différentes options pour les familles et les praticiens afin de prévenir la morbidité, la mortalité ou la douleur et d'améliorer globalement les résultats obstétricaux et néonataux (Ricciotti, Chen et Sachs, 1995; Dugas, 2011). On peut penser au dépistage prénatal (Bekker, Hewison et Thornton, 2004), au mode d'accouchement après une césarienne (Frost, et collab., 2009), aux options lors des présentations par le siège (Nassar, et collab., 2007), aux interruptions de grossesse (Wong, et collab., 2006) ou à l'utilisation de la péridurale (Stewart, et collab., 2003). Ces différentes possibilités de gestion de la grossesse et de l'accouchement représentent cependant autant de prises de décision, de dilemmes, auxquels de nombreux parents pourraient être confrontés.

Afin de procéder au choix des options de soins obstétricaux, le modèle de partage de la décision médicale par lequel les cliniciens et les femmes — accompagnées ou non de leurs proches - 
"L'augmentation significative du degré d'implication des femmes dans les décisions médicales et la prééminence du consentement éclairé a émergé en réaction aux préoccupations éthiques et médicolégales, associées aux options offertes dans le cadre d'interventions obstétricales, et à la tangente prise par le système de santé canadien pour une offre de soins axée sur le patient..." discutent des risques et avantages des différentes options, révèlent leurs préférences de traitement et en arrivent à une décision conjointe constitue actuellement le principal paradigme pour les guider dans le choix des différentes interventions (Dodd, et collab., 2007; Deutchman et Roberts, 2003; Kenton, et collab., 2005). L'augmentation significative du degré d'implication des femmes dans les décisions médicales et la prééminence du consentement éclairé a émergé en réaction aux préoccupations éthiques et médico-légales, associées aux options offertes dans le cadre d'interventions obstétricales, et à la tangente prise par le système de santé canadien pour une offre de soins axée sur le patient (Deutchman et Roberts, 2003; Dugas et Graham, 2011; Kenton, et collab., 2005; American Congress of Obstetricians and Gynecologists, 2010; Caughey, 2010; Roberts, et collab., 1997).

Bien que tout à fait louable, cette orientation de l'approche médicale vis-à-vis du patient pose un certain nombre de défis et d'obstacles qu'il faudra très bien gérer afin que l'expérience de celui à qui l'on demande de s'impliquer dans la décision soit positive. En effet, pour éviter que ce modèle de soins soit source de stress ou d'anxiété chez le patient, il importe que ce dernier soit très bien informé, qu'il ait les connaissances requises pour s'impliquer dans la prise de décision et pour bien évaluer les options.

Concrètement, une information claire et complète concernant les risques et les avantages doit être fournie, bien comprise et soupesée de façon à ce que le patient puisse prendre une décision éclairée entre les différentes options qui lui sont présentées (Bekker, Hewison et Thornton, 2004; Société des obstétriciens et gynécologues du Canada, 2005; Hannah, et collab., 1986). Ici, la qualité des conseils médicaux et de la provision d'information est intimement liée à la satisfaction du patient et à la qualité perçue des soins (Dodd, et collab., 2007; Deutchman et Roberts, 2003; Kenton, et collab., 2005; Roberts, et collab., 1997; Nassar, et collab., 2007; Chaillet, et collab., 2007; 2006).

Toutefois, le choix du patient et la satisfaction qui en découle ne sont pas seulement liés à l'apport d'information au sujet des risques et des avantages des différentes options, mais dépendent aussi des expériences passées, des besoins personnels préexistants, 
"Le patient à qui l'on demande de s'impliquer et de prendre une décision doit être efficacement guidé dans le processus de prise de décision."

\section{"Prenant pour} exemple le processus de décision concernant le mode d'accouchement après césarienne, nous allons explorer quelques aspects $d u$ processus de prise de décision partagée en obstétrique. » des valeurs et des motivations qui lui sont propres (Dodd et collab., 2007; Deutchman et Roberts, 2003; Roberts, et collab., 1997; Goldman, et collab., 1990; Paré, Quinones et Macones, 2006). Le patient à qui l'on demande de s'impliquer et de prendre une décision doit être efficacement guidé dans le processus de prise de décision. On doit pouvoir l'encadrer pour lui permettre d'examiner les avantages et les inconvénients de chacune des options, tout en l'aidant à bien cerner ses propres valeurs et motivations. En effet, en l'absence d'adéquation entre la décision prise et les valeurs, attentes ou motivations, des chercheurs ont noté que de l'anxiété et une forme de conflit décisionnel peuvent apparaitre et avoir un impact négatif direct sur les issues physiques et psychologiques de santé (Glazier, et collab., 2004).

Prenant pour exemple le processus de décision concernant le mode d'accouchement après césarienne, nous allons explorer quelques aspects du processus de prise de décision partagée en obstétrique. Tenter un accouchement vaginal après césarienne (AVAC)? Programmer une deuxième césarienne? Les études les plus récentes démontrent que les deux options présentent chacune leur part de risques et de bénéfices qu'il convient de considérer avant d'opter pour l'une ou l'autre (American Congress of Obstetricians and Gynecologists, 2010; Caughey, 2010; Roberts, et collab., 1997; Kenton, et collab., 2005). Alors que la rupture utérine constitue un risque majeur lors d'un essai d'AVAC, la césarienne itérative, en tant que chirurgie, n'est pas sans risque non plus (American Congress of Obstetricians and Gynecologists, 2010; Paré, Quinones et Macones, 2006). Le choix du mode d'accouchement après césarienne est donc un cas obstétrical type pour lequel il convient, dans une perspective de respect éthique, d'opter pour le modèle de prise de décision partagée (Dodd, et collab., 2007; Deutchman et Roberts, 2003; Paré, Quinones et Macones, 2006; Roberts, et collab., 1997). Et bien que le conjoint ou d'autres membres de sa famille soient régulièrement impliqués dans le processus de prise de décision, le choix final revient, dans un souci éthique évident, à la femme seule, puisque c'est elle qui aura à subir les conséquences physiques ou psychologiques liées à son choix de mode d'accouchement. 


\section{La méthodologie}

"L'objectif principal de cette étude était de permettre le développement et l'évaluation d'un outil d'aide à la décision adapté à leur besoin.»
La recherche s'est déroulée au Centre hospitalier universitaire Sainte-Justine (CHU Sainte-Justine), un hôpital de troisième ligne qui est actuellement le plus grand centre mère-enfant au Canada. En 2007, il a reçu le statut d'" hôpital promoteur de la santé » par l'OMS, c'est-à-dire qu'on s'y attarde à développer un ensemble de stratégies qui permettent aux hôpitaux de mieux s'adapter aux changements cliniques ou sociaux et de s'impliquer dans la promotion de la santé des patients, du personnel et de la communauté. Le CHU Sainte-Justine voit l'importance de diminuer toute intervention invasive ou chirurgicale lors de la grossesse et de l'accouchement (évitement de la césarienne de convenance, de l'épisiotomie, de l'induction) au profit d'une approche plus humaine de la naissance et des soins à l'enfant, par la promotion du modèle de prise de décision partagée, par la promotion de l'allaitement ou encore par la valorisation de l'accompagnement personnalisé à la naissance.

Les données recueillies au CHU Sainte-Justine permettront d'illustrer en contexte obstétrical l'application de ce modèle de prise de décision partagée. Elles proviennent d'une enquête qualitative réalisée en juillet et en août 2011 auprès de femmes ayant vécu ce processus de prise de décision pour leur mode d'accouchement après césarienne. L'objectif principal de cette étude était de permettre le développement et l'évaluation d'un outil d'aide à la décision adapté à leur besoin.

Vingt femmes de diverses origines ethniques ont été rencontrées après leur accouchement pour un entretien semi-dirigé, lors de leur séjour en post-partum au CHU Sainte-Justine. Ces participantes avaient bénéficié d'un suivi obstétrical dans différentes cliniques ou hôpitaux de la grande région de Montréal. Elles provenaient de différentes nationalités : québécoise (six), haïtienne (quatre), française (deux), algérienne (deux), guinéenne (une), rwandaise (une), cambodgienne (une), mexicaine (une), colombienne (une). Onze d'entre elles avaient choisi la césarienne et neuf avaient opté pour l'AVAC. Aucune sélection en fonction 
des caractéristiques socioculturelles n'a été réalisée pour cette étude. Les vingt premières femmes à être approchées pour l'étude, qui venaient d'accoucher et qui étaient en séjour post-partum à l'hôpital, ont accepté généreusement de répondre à nos questions portant sur leur expérience personnelle. La variabilité rencontrée est donc relativement représentative de la réalité de cet hôpital.

Nous avons demandé à ces participantes de nous parler de ce processus de prise de décision. Nous leur avons d'abord demandé de nous décrire le rôle qu'elles ont joué dans le processus de décision et le degré de soutien reçu des professionnels de la santé et de leurs proches. Nous leur avons ensuite demandé de nous parler de l'information qu'elles ont reçue - nature et provenance - pour en arriver à une décision. Puis, nous les avons interrogées sur le niveau d'anxiété qu'elles ont pu ressentir alors qu'elles devaient prendre cette importante décision. Enfin, nous leur avons demandé de nous dévoiler leur préférence initiale pour l'un ou l'autre des modes d'accouchement et les raisons qui ont motivé leur décision.

Après avoir décrit le processus décisionnel tel qu'exprimé par les participantes à notre enquête, nous verrons quels outils fréquemment utilisés dans le contexte obstétrical pourraient permettre de faciliter la tâche aux femmes qui se retrouvent en pareille situation.

\section{Le rôle dans la prise de décision et le degré de soutien reçu}

Le rapport patient-médecin a beaucoup évolué ces dernières décennies. Le modèle paternaliste s'est estompé pour faire place à un modèle de prise de décision partagée, lequel est désormais incontestable puisque, depuis son déploiement, il a démontré de nombreux bénéfices tant pour les patients, telles les femmes en suivi obstétrical, que pour les consultants (Twisselmann, 2006). Parmi les principaux bénéfices du modèle de prise de décision partagée, nous retrouvons : une satisfaction accrue des soins 
"Parmi les principaux bénéfices du modèle de prise de décision partagée, nous retrouvons : une satisfaction accrue des soins ou des traitements reçus puisqu'ils répondent davantage aux différentes motivations du patient..." ou des traitements reçus puisqu'ils répondent davantage aux différentes motivations du patient; le sentiment chez ce dernier d'avoir plus de contrôle sur sa maladie, son état ou ses traitements; une amélioration de la qualité de vie grâce à des soins ou des traitements adaptés aux particularités du patient; une meilleure compréhension de la maladie ou de l'état particulier du patient et des options de traitement; une diminution de la souffrance liée aux complications éventuelles; une amélioration de la capacité fonctionnelle; une atténuation des craintes; ainsi qu'une meilleure compliance thérapeutique (Twisselmann, 2006).

De plus, en vertu des principes éthiques qui gèrent notre système de santé, dont le principe d'autonomie qui accorde le droit de regard du patient sur les soins de santé qui lui seront dispensés, nous croyons que ce modèle de prise de décision partagée patient-médecin doit bénéficier de toute l'attention possible, afin d'en corriger les faiblesses et d'en baliser l'application. C'est la raison pour laquelle il nous semblait essentiel de demander aux participantes de décrire le rôle qu'elles ont joué dans le processus de décision et le degré de soutien qu'elles ont reçu de la part des professionnels de la santé et de leurs proches.

Nous leur avons demandé de nous dire si, selon elles, leur mode d'accouchement après césarienne était plutôt leur décision, une décision prise de concert avec leur médecin ou encore, une décision du médecin seul. Nous leur avons aussi demandé de commenter le degré de soutien qu'elles ont reçu de la part de leur médecin, de même que de leurs proches, pendant le processus de prise de décision.

Sur les dix-huit participantes qui ont répondu à la première question, seules cinq ont affirmé qu'il s'agissait d'une décision prise de façon partagée avec leur médecin et onze considéraient qu'il s'agissait plutôt de leur propre décision et que le médecin n'y avait joué aucun rôle, si ce n'est de s'assurer de leur éligibilité à l'AVAC. Enfin, deux participantes ont affirmé que la décision venait davantage au médecin.

Sur les dix-sept répondantes qui se sont prononcées sur le soutien de leur médecin, quatorze ( $82 \%$ ) se sont dites très 
"Il est intéressant de noter à la lumière de ces chiffres une tendance des participantes à se sentir globalement soutenues par leur médecin..." "...c'est bien souvent le soutien du conjoint qui était le plus important. » satisfaites du degré de soutien de sa part. Et treize répondantes (81\%) sur seize ont affirmé avoir reçu un très bon soutien de la part de leurs proches. Enfin, trois répondantes ont affirmé n'avoir reçu qu'un faible soutien de la part de leur médecin et trois autres, différentes des premières, ont affirmé n'avoir reçu qu'un faible soutien de la part de leurs proches.

Il est intéressant de noter à la lumière de ces chiffres une tendance des participantes à se sentir globalement soutenues par leur médecin, malgré le fait que, dans l'ensemble (72\% des cas), leur prise de décision n'ait pas été réalisée de concert avec ce dernier comme le prévoit le modèle de prise de décision partagée. Dans certains cas, bien qu'elles se soient senties globalement satisfaites du degré de soutien, certaines participantes auraient a priori souhaité une plus grande implication du médecin. Une participante explique :

«C'est certain que j'aurais presque voulu qu' elle me dise quoi faire. Quand je suis sortie du bureau la première fois, j'étais déçue qu'elle ne me dise pas : "regarde, dans ton cas, moi je pense que je ferais telle chose." Je lui ai dit : "tu sais je trouve ça plate, j'ai l'impression que tu me donnes la liste [des risques et bénéfices], mais qu'après..." Elle m'a dit : "tu sais, moi je ne peux pas vivre avec la conséquence de t'avoir dit de faire ça, parce que je ne connais pas la suite des choses. Mais je peux t'éclairer, t'expliquer, puis après tu fais ton choix". Donc quand j'ai compris ça, je me suis dit : dans le fond, j'ai été bien guidée, elle a répondu à mes questions. Puis elle ne m'a pas jugée quand j'ai choisi la césarienne. J'avais peur d'être jugée, tu sais, dans le sens : "elle prend la voie facile". Mais pas du tout. Elle m'accompagnait là-dedans. » (Participante ayant choisi la césarienne)

Concernant le soutien des proches, c'est bien souvent le soutien du conjoint qui était le plus important. Concernant les 
autres membres de la famille et amis, bien que leur soutien leur semblât suffisant à leurs dires, plusieurs des répondantes ont aussi affirmé avoir ressenti venant d'eux de la pression en faveur de l'une ou de l'autre option, ce qu'elles n'avaient pas ressenti ni de la part de leur médecin, ni de la part de leur conjoint :

"Mon mari, c'était excellent. Mais, mes parents, ma sœur qui a eu une césarienne pour ses jumeaux, pour eux c'était clair que [l'AVAC n'était pas une bonne idée]. Mon père espérait que je finisse en césarienne pour mon premier garçon. L'accouchement lui faisait peur. Détresse fœetale, anévrisme de la maman, il voyait seulement des drames. Ma mère m'a appelée quelques jours avant que mon travail commence pour me dire : "Mais là, tu es certaine que c'est une bonne idée d'accoucher?" J'ai parlé à des gens qui disaient que, comme j'avais déjà eu une césarienne, une autre césarienne serait plus facile pour moi. Non, mes proches ne m'ont pas du tout soutenue, à part mon mari. » (Participante ayant choisi l'AVAC)

Dans le même esprit, une autre participante raconte :

"Pour moi la personne la plus importante et de qui je voulais avoir du soutien était mon mari, plus que les autres. L'ignorance des gens crée beaucoup de craintes et ils veulent te transmettre leurs craintes. Je n'ai pas évité de consulter mes proches, mais moi, j’ai vécu ça [la prise de décision] avec mon mari. C'est lui que j'ai consulté vraiment. " (Participante ayant choisi l'AVAC)

Dans ces différents témoignages, le facteur "information " revient. D'abord dans le premier cas où la participante se dit satisfaite de l'information reçue de son médecin, puis dans les deux autres cas, un manque d'information de la part des proches qui 
voyaient dans l'accouchement vaginal des risques démesurés par rapport à la césarienne qu'ils considéraient comme plus sécuritaire. Or, la littérature indique que lorsque la femme est éligible à l'AVAC, c'est cette option qui représente le risque de morbidité le plus faible tant pour la mère que pour l'enfant (Société des obstétriciens et gynécologues du Canada, 2005).

\section{Information reçue et prise de décision}

"...l'hétérogénéité de l'information de masse disponible aux patientes et les variations des pratiques médicales peuvent rendre plus difficile la prise de décision..."
Le transfert de connaissances constitue donc un important facteur dans la prise de décision partagée. À cet effet, il est recommandé qu'une information adéquate soit systématiquement fournie afin que les femmes éligibles à un AVAC puissent choisir de façon éclairée entre la césarienne et l'essai de travail (American Congress of Obstetricians and Gynecologists, 2010; Société des obstétriciens et gynécologues du Canada, 2005).

Cependant, comme nous venons de le voir, le processus de décision peut être difficile pour la mère dû à l'hétérogénéité de l'information disponible (Dodd, et collab., 2007; Deutchman et Roberts, 2003; Kenton, et collab., 2005; Montgomery, et collab., 2007). En effet, l'hétérogénéité de l'information de masse disponible aux patientes et les variations des pratiques médicales peuvent rendre plus difficile la prise de décision (Caughey, 2010; Hannah, et collab., 1986; Iglesias, Burn et Saunders, 1991). Le manque de temps pour des séances d'information avec les familles constitue aussi une importante problématique (Iglesias, Burn et Saunders, 1991; Glazier, et collab., 2004).

Afin d'en connaitre la nature et la qualité perçue, nous avons demandé aux participantes leur avis sur la quantité d'information reçue lors de consultations. Puis, nous leur avons demandé si elles avaient eu accès à d'autres sources d'information pour prendre une décision éclairée quant à leur mode d'accouchement après césarienne et si elles avaient consulté à cet effet le web ou des forums de discussion.

Onze participantes (69\%) sur les seize qui se sont prononcées sur le sujet se sont déclarées satisfaites de la quantité d'information 
"Le manque de documentation remise aux mères pour être apportée et lue à la maison, plus que le manque d'information fournie par le médecin, peut expliquer en partie le recours à internet pour la recherche d'information complémentaire. " reçue, même si au moment de l'entretien la plupart ne se souvenaient que de quelques bribes d'information, comme la rupture utérine (risque de l'AVAC) ou les risques liés à toute chirurgie (risques de la césarienne). Cinq participantes (28\%) sur les dix-huit qui ont répondu à cette question ont eu accès à de la documentation complémentaire qu'elles ont elles-mêmes recherchée et dix participantes (56\%) ont trouvé de l'information sur le web ou dans des forums de discussion.

Ce qu'il faut noter ici, c'est que le tiers des participantes se sont dites insatisfaites de l'information reçue, et ce, pour différentes raisons, et que plus de la moitié d'entre elles sont allées chercher de l'information sur le web. Le manque de documentation remise aux mères pour être apportée et lue à la maison, plus que le manque d'information fournie par le médecin, peut expliquer en partie le recours à internet pour la recherche d'information complémentaire.

Une participante se disant quelque peu insatisfaite de la quantité d'information reçue explique :

"Je n'ai pas reçu de renseignements sur la césarienne parce que ce n'était pas le sujet à traiter avec le médecin. J'avais choisi l'accouchement vaginal, alors $\mathrm{j}$ 'ai reçu des informations sur l'AVAC. Peut-être que j'aurais aimé avoir plus de détails, entrer plus en profondeur avec les risques de l'AVAC. J'aurais aimé avoir quelques paperasses à lire, comme ça. Même une séance d'information sur les risques, parce que je voulais être prête à tout. Je ne voulais pas être surprise comme pour mon premier accouchement. Je n'étais pas préparée pour une césarienne. Par exemple, si j'avais eu une rupture utérine, des choses comme ça... En gros, j'aurais aimé une information plus complète.» (Participante ayant choisi l'AVAC) 


\section{«Un élément}

important qui est revenu à plusieurs reprises lors de nos entretiens relatifs à l'information était leur besoin de se sentir informées et préparées pour toute éventualité. »
Concernant le recours à internet, une autre participante explique :

«[Sur les forums de discussion, je ne discutais] pas nécessairement avec celles qui avaient eu un AVAC ou une césarienne, mais avec des femmes enceintes, et je leur demandais leur opinion. Il y en a certaines qui avaient eu une césarienne antérieure et puis, pour elles, c'était clair qu'elles tentaient l'AVAC. Clair, clair, clair! Puis on était une petite bande d'ailleurs. Puis, jusqu'à maintenant, on l'a toutes réussi! Ça m'a aidée parce qu'on entend tellement toute sorte d'opinions à gauche, à droite. " (Participante ayant choisi l'AVAC)

Avant de prendre une décision, une majorité de participantes a donc cherché à compléter l'information reçue en consultation par le recours à d'autres sources d'information. Un élément important qui est revenu à plusieurs reprises lors de nos entretiens relatifs à l'information était leur besoin de se sentir informées et préparées pour toute éventualité. Pour certaines, l'inconnu a d'ailleurs été une importante source d'anxiété lors du processus décisionnel.

\section{L'anxiété liée au processus de prise de décision}

L'état d'anxiété et le sentiment de conflit décisionnel peuvent résulter du processus de prise de décision lorsque l'information n'est pas jugée suffisante, lorsqu'il y a un manque de soutien dans la prise de décision ou encore lorsque les valeurs ne sont pas respectées ou que les attentes ne sont pas satisfaites (Kenton, et collab., 2005; Roberts, et collab., 1997; Chaillet, et collab., 2006; 2007; Goldman, et collab., 1990; Montgomery, et collab., 2007). Ces états psychologiques lors de la grossesse et de l'accouchement peuvent augmenter le risque de résultats de santé défavorables pour les mères et les nouveau-nés, réduisant du coup la satisfaction globale et la qualité perçue des soins de santé (Glazier, et collab., 2004). 
"...au moment de la grossesse, plusieurs femmes ressentent $d u$ stress ou de la peur à l'approche de la date de l'accouchement, d'autant plus lorsque le précédent s'est terminé en césarienne d'urgence. "
L'anxiété peut être définie comme étant le sentiment de nervosité ou de tension psychologique ou physique découlant de l'anticipation des événements négatifs liés au choix (O'Connor et Jacobsen, 2000). Le conflit décisionnel peut être défini comme étant l'incertitude quant à la ligne de conduite à adopter lorsque le choix entre des options implique un risque, une perte ou une remise en question des valeurs personnelles (O'Connor et Jacobsen, 2000). Ainsi, en obstétrique, ce processus de prise de décision est particulièrement délicat, puisque la décision à prendre est souvent chargée d'émotion. La femme, accompagnée de ses proches, doit décider pour sa propre santé, mais aussi pour celle de son enfant. De plus, au moment de la grossesse, plusieurs femmes ressentent du stress ou de la peur à l'approche de la date de l'accouchement, d'autant plus lorsque le précédent s'est terminé en césarienne d'urgence. Lorsqu'en plus, on demande à la femme de décider de son mode d'accouchement, il peut en résulter une grande anxiété.

Afin de mesurer l'anxiété ressentie par les participantes au moment de prendre leur décision sur leur mode d'accouchement, nous avons utilisé un questionnaire inspiré de Spielberger selon la version abrégée de Marteau et Bekker (1992). Les résultats nous ont donné une cote d'anxiété que nous n'utiliserons qu'à titre indicatif, puisque les conditions normales d'administration de ce type de questionnaire ne pouvaient être respectées dans le cadre de la présente étude.Avec une cote de 14 à 17, le niveau d'anxiété peut être considéré comme présent, mais modéré.Au-delà de 17, le niveau d'anxiété devient élevé.

Parmi les dix-sept participantes qui ont rempli ce questionnaire, plus de la moitié (53\%) ont décrit un état modérément anxieux (six participantes) ou très anxieux (trois participantes).

De plus, au cours des entretiens, certaines ont exprimé spontanément des états émotionnels pouvant être liés à cette anxiété :

"Je pensais au départ que je n'avais pas le choix d'avoir une césarienne. Comme j'en avais déjà eu une, il y avait comme une croyance que je n'avais pas le choix. Puis, c'est en parlant à 
mon entourage : "non, non $[\ldots]$ tu peux faire un choix, décider de toi-même un AVAC." Là, j'ai été conscientisée dès le début de ma grossesse que c'était une option d'accoucher naturellement. Mais, là, plus la grossesse avançait, moins ça me tentait d'avoir un AVAC. Tu sais, chacun gère son niveau de risque dans la vie, puis plus ça allait, plus je me disais :"est-ce que j'ai envie de prendre ce risque, pour moi, pour ma famille? J'ai un autre enfant..." Et plus ça allait, plus j'angoissais à l'idée. Puis, je me disais : "si j'accouche naturellement, puis que je suis angoissée comme ça, ça risque de tourner mal.” " (Participante ayant choisi la césarienne)

Une autre participante nous raconte :

"Je suis contente d'avoir eu un accouchement vaginal, que ça ait réussi, que mon bébé soit en santé, que tout se soit bien passé. Ça me stressait... Pour la césarienne, le premier bébé,je n'étais pas stressée, mais lui [le deuxième], j'étais stressée, parce que j'avais des risques d'avoir une rupture utérine. Je n'étais pas stressée d'avoir peut-être une césarienne, mais j'étais stressée d'avoir une rupture utérine ou un problème au bébé.» (Participante ayant choisi l'AVAC)

Certaines participantes ont avoué avoir choisi une option plutôt qu'une autre, non pas en fonction d'une comparaison rationnelle des risques et bénéfices de chacune, mais plutôt en fonction de leur niveau de stress ou d'anxiété face à une conséquence possible de leur choix.

\section{Préférence initiale et décision ultime}

Dans le cas des femmes ayant un antécédent de césarienne, le choix du mode d'accouchement dépend de la perception des 
risques associés à l'une ou à l'autre des options, soit la césarienne élective ou l'essai de travail.

Nous le disions en introduction, le choix pour une femme ou des parents de tenter ou non une intervention et la satisfaction qui en découle dépendent grandement des expériences passées, des besoins personnels, des valeurs et des attentes propres à chacun (Dodd, et collab., 2007; Deutchman et Roberts, 2003; Roberts, et collab.,1997; Goldman, et collab., 1990; Paré, Quinones et Macones, 2006). Parmi les facteurs les plus cités dans le domaine obstétrical nous retrouvons : les craintes de la douleur, de la chirurgie ou de vivre une césarienne d'urgence (Roberts, et collab., 1997; Iglesias, Burn et Saunders, 1991); les soucis de l'impact de l'intervention sur le bébé (Roberts, et collab., 1997; Iglesias, Burn et Saunders, 1991); les appréhensions créées par l'expérience d'une naissance précédente ou le désir de grossesse future (Dodd, et collab., 2007; Deutchman et Roberts, 2003; Goldman, et collab., 1990); et les besoins en termes de chirurgie additionnelle ou de planification familiale (Deutchman et Roberts, 2003; Roberts, et collab., 1997; Paré, Quinones et Macones, 2006).

Dans notre échantillon, trois participantes préféraient la césarienne au début de leur grossesse. Leurs principales raisons étaient : les risques de rupture utérine (une participante); le sentiment de sécurité avec la césarienne (deux participantes); et la peur de l'accouchement (une participante).

Toujours dans notre échantillon, treize participantes préféraient l'accouchement vaginal au début de leur grossesse. Les raisons évoquées étaient : une récupération plus rapide (huit participantes); le désir de vivre l'expérience d'un accouchement vaginal (cinq participantes); le désir d'un accouchement plus naturel (quatre participantes); le désir d'un séjour plus court à l'hôpital (trois participantes); le désir d'éviter une nouvelle chirurgie (deux participantes); la volonté d'augmenter les chances de pouvoir allaiter (deux participantes); la volonté de pouvoir s'occuper de son autre enfant à la maison et de pouvoir le soulever (deux participantes); la volonté d'éviter les complications des cicatrices pour les prochains accouchements (deux participantes); et le désir d'éviter la prise de médicament après l'accouchement (une participante). 
Enfin, la raison de l'abstention de deux participantes de se prononcer sur leur mode d'accouchement en début de grossesse était la peur de revivre le scénario du premier accouchement et d'avoir une autre césarienne d'urgence. Elles préféraient donc voir si les raisons de la première césarienne pouvaient se présenter à nouveau avant de réfléchir sur le mode d'accouchement.

Parmi les cinq participantes qui, en début de grossesse, désiraient un accouchement vaginal et qui ont finalement eu une césarienne, les raisons étaient : une absence de déclenchement naturel du travail en fin de grossesse (deux participantes); un problème d'hypertension (une participante); un refus d'AVAC à cause de la peur de la rupture utérine (une participante); et un échec d'AVAC (une participante).

Ce sont donc cinq participantes (38\%) sur treize qui n'ont pas eu le type d'accouchement qu'elles souhaitaient au départ. D'où l'importance d'obtenir toute l'information, à la fois sur leur choix premier, mais aussi sur la deuxième option, car aucune d'entre elles ne pouvait prévoir l'issue de leur grossesse. Dans certains cas, cette expérience peut même être très difficile. Une participante qui souhaitait un AVAC, mais qui a accouché par césarienne nous relate son expérience :

" [Pour le premier bébé], j'avais eu 8 mois de grossesse extraordinaires. À aucun moment je ne m'étais dit : "je vais peut-être avoir une césarienne". J'ai mis du temps à me dire que j'avais accouché... Je n'arrivais pas à me dire que j'avais accouché. C'était plutôt traumatisant le lendemain quand on m'a expliqué le caractère urgent de la césarienne. J'ai alors compris un peu ce qui s'est passé, et ça a été, oui, un traumatisme. J'ai mis beaucoup de temps à m'en remettre. [Pour le deuxième] je pensais qu'elle allait venir naturellement. Bien là, le fait que ce soit programmé, ça m'a fait bizarre moi. Ça me paraissait surréaliste. De me dire que tel jour, si elle n'était pas venue toute seule, il y aurait 
«Bien préparer les femmes à toutes les possibilités dès le début de leur grossesse s'avère d'une grande importance. » une césarienne pour éviter des complications par la suite. Ça me paraissait complètement surréaliste. » (Participante ayant choisi l'AVAC, mais qui a accouché par césarienne)

Bien préparer les femmes à toutes les possibilités dès le début de leur grossesse s'avère d'une grande importance. Cette préparation à la prise de décision peut être facilitée en obstétrique grâce à différents outils validés à cet effet.

\section{Les outils pour faciliter la prise de décision}

"Les outils

d'information, sous

forme d'imprimés

ou sur support

informatique, sont

efficaces..."
Différents outils visant à faciliter la consultation médicale, le partage de l'information et le processus de prise de décision sont régulièrement utilisés en obstétrique : les outils d'information par écrit (supports imprimés ou informatiques); les consultations ciblées (individuelles ou en groupe); l'arbre de décision (algorithme) et les outils d'analyse décisionnelle (supports imprimés ou informatiques).

Les outils d'information, sous forme d'imprimés ou sur support informatique, sont efficaces pour fournir aux parents de l'information générale sur les différentes options qui peuvent se présenter à eux au cours d'une grossesse (Glazier, et collab., 1997; Hunter, et collab., 2005). Toutefois, ce genre d'outil doit être utilisé en complément d'explications de la part des prestataires de services dans le cadre des soins habituels et n'agit pas sur le conflit décisionnel ou sur l'anxiété qui l'accompagne (Frost, et collab., 2009; Wong, et collab., 2006; Stewart, et collab., 2003; Glazier, et collab., 1997). De plus, d'importantes variations socioculturelles persistent relativement aux outils sur support informatique ou à la compétence à les utiliser. Connaissant la grande variabilité socioculturelle de notre population de femmes enceintes et dans un souci de répondre aux besoins de la majorité d'entre elles, le type d'outils sur support informatique devrait être évité, dans la mesure du possible. 
"Les outils d'analyse décisionnelle sont efficaces pour le transfert de connaissances, la réduction de l'anxiété, la satisfaction de la décision, la réduction des conflits décisionnels (sécurité) et sur le choix final..."
L'arbre de décision est une structure visuelle, pourvue de branches successives, qui décrit la séquence d'événements et de résultats possibles pour progressivement orienter le patient vers un choix en fonction de ses valeurs à chacun des nœuds de décision (Thomson, et collab., 2006). Cependant, l'arbre de décision n'est pas efficace en ce qui concerne le transfert de connaissances, le conflit décisionnel ou l'anxiété.

Les consultations individuelles ou en groupe permettent au patient de recevoir de l'information détaillée et personnalisée sur les options de soins et, puisqu'elles se présentent sous forme de dialogues de vive voix, elles lui permettent de recevoir les réponses qui sauront le rassurer sur les choix auxquels il est confronté (DeLucia-Waack, et collab., 2004). Les consultations individuelles ou en groupe permettent aussi aux participants d'apprivoiser une situation médicale donnée et les moyens de faire face à l'anxiété au cours de leur processus décisionnel (Fraser, et collab., 1997). Un avantage important de la consultation individuelle est l'interaction approfondie, ciblée et directe patient-médecin (Hunter, et collab., 2005). Par contre, les consultations en groupe ne sont pas efficaces dans la prévention du conflit décisionnel. De plus, la variabilité de la composition des groupes de soutien résultant de la variabilité socioculturelle présente dans notre population de femmes enceintes peut rendre plus difficiles à prédire les résultats de ce type d'outil d'aide à la décision. Le modèle de consultation individuelle devrait donc être privilégié lorsque la population est plutôt multiethnique.

Les outils d'analyse décisionnelle comportent plusieurs volets : une présentation des choix de traitement; une description et une représentation visuelle des risques et des avantages de chaque option; une méthode pour aider les patients à clarifier leurs valeurs personnelles face à chacune des options et à leurs conséquences respectives; et une méthode les aidant à reconnaitre les avantages et les inconvénients qui sont les plus importants à leurs yeux. Les outils d'analyse décisionnelle permettent de passer efficacement par les différentes étapes du processus de prise de décision (O'Connor, et collab., 2003) pour en arriver à une décision personnalisée (Wong, et collab., 2006). Ce moyen 
sert aux professionnels de la santé en fournissant de l'information basée sur les preuves (evidence-based) et sur les options de soins. Les outils d'analyse décisionnelle sont efficaces pour le transfert de connaissances, la réduction de l'anxiété, la satisfaction de la décision, la réduction des conflits décisionnels (sécurité) et sur le choix final (Nassar, et collab., 2007).

Concernant la pertinence de l'utilisation d'outils, une participante commente :

«Tu sais, si j'avais eu plus d'outils, faciles, parce que, tu sais, la vie, ça va vite. On travaille, puis on a des enfants, puis on n'a pas nécessairement le temps. Puis, tu sais, le fait que j'ai dû moi-même choisir... Puis en même temps, un rendez-vous avec ton médecin, ça ne dure pas une heure, ça dure dix minutes. Alors, je comprends qu'en dix minutes, on ne peut pas m'expliquer toute la gamme d'avantages et d'inconvénients. Puis me dire des histoires vécues, et tout... Au début, l'AVAC, c'était une option. Si j'avais eu un outil quelconque, dans lequel on m'aurait indiqué des sites internet, des lectures, des histoires à succès, je ne sais pas, mais il me semble que ça aurait été un guide.» (Participante ayant finalement choisi la césarienne)

Ces outils, et en particulier les outils d'analyse décisionnelle, auraient donc pu répondre aux besoins de cette participante et peut-être la guider vers le choix d'AVAC, qui est recommandé entre autres par la SOGC dans les situations où la femme est éligible à l'essai de travail par voie vaginale.

Enfin, les participantes à notre étude provenaient de neuf horizons culturels différents, mais toutes semblaient avoir vécu plus ou moins la même situation. Cela suggère qu'un seul outil, s'il est adapté, peut convenir aux besoins de la plupart des parents et augmenter sensiblement leur satisfaction des soins reçus. Une étude plus pointue portant sur les besoins particuliers de chacune des participantes aurait pu révéler davantage de spécificité 
culturelle que nous en avons trouvé dans le cadre de notre travail. Une telle étude plus ciblée n'entrait pas dans le cadre du présent travail, son objectif principal étant de permettre le développement d'un outil d'aide à la décision qui soit cliniquement et facilement applicable à une majorité de femmes.

\section{Conclusion}

"Le défi qui se pose ici est donc d'arriver à impliquer davantage les médecins, à les amener en quelque sorte dans la prise de décision..."

"Il semble en effet inconvenant de demander aux femmes de choisir leur mode d'accouchement alors qu'elles ne se sentent pas adéquatement informées. "
Dans cet article, nous avons tenté d'illustrer par divers exemples comment se présente le modèle de prise de décision partagée en contexte obstétrical, plus particulièrement en ce qui concerne le mode d'accouchement après césarienne. De façon générale, on peut voir que ce dernier procède davantage du principe d'autonomie qui accorde le droit de regard du patient sur les soins qui lui seront administrés que d'un partage de la décision médicale, par lequel les cliniciens et les femmes discuteraient de risques et d'avantages et, conjointement, prendraient une décision. Le défi qui se pose ici est donc d'arriver à impliquer davantage les médecins, à les amener en quelque sorte dans la prise de décision, afin d'éviter que certaines femmes, pour qui ce modèle d'autonomie ne conviendrait pas, se sentent seules ou délaissées par leur médecin dans ce processus décisionnel au nom de l'éthique ou de considérations purement médico-légales. Dans ce cas de figure, le fait de simplement remettre à la femme un outil d'aide à la décision ne suffit pas.

En ce qui concerne le transfert de connaissances sur les risques et bénéfices des deux options d'accouchement, certaines participantes se sont dites insatisfaites de l'information reçue lors de consultations. Elles ont cherché d'autres sources d'information, que ce soit via les livres ou sur internet.Vu la qualité inégale de l'information véhiculée dans ces autres sources, un besoin s'impose de fournir en complément à l'information fournie par le médecin des renseignements fiables, sous forme de dépliants, sur internet, lors de consultations individuelles ou de groupe ou par l'entremise d'outils d'analyse décisionnelle. Il semble en effet inconvenant de demander aux femmes de choisir leur mode 
d'accouchement alors qu'elles ne se sentent pas adéquatement informées. Dans ces conditions, le choix du mode d'accouchement risque de s'effectuer en fonction de vagues impressions sur les risques et bénéfices des différentes options, ou sur des idées reçues comme dans le fameux dicton "césarienne un jour, césarienne toujours " qui prévalait encore jusqu'à très récemment. Par contre, afin d'éviter de mettre davantage de pression sur les femmes engagées dans une prise de décision, l'information qui leur est fournie par l'entremise de l'un ou de l'autre de ces outils se doit impérativement d'être neutre et d'exposer honnêtement les faits relatifs aux risques et bénéfices de chacune des options, plutôt que de tenter d'imposer une idéologie médicale.

Enfin, l'anxiété remarquée chez bon nombre de participantes devant la décision à prendre apparaît aussi comme objet de préoccupation. Ici, ce sont les consultations individuelles ou de groupe de même que les outils d'analyse décisionnelle qui semblent les plus efficaces pour gérer l'anxiété des futures mères. Ces outils servent aussi à faciliter leur prise de décision et à augmenter leur satisfaction générale des soins reçus, tant du point de vue de l'information qui leur a été transmise que d'un encadrement conduisant à la meilleure option qui soit, pour

"Il est en effet inadmissible que des pratiques qui se veulent favorables au patient, comme son implication dans la prise de décision, puissent générer chez lui de la détresse psychologique, comme l'ont exprimé les participantes à notre étude. » elles-mêmes et pour leur enfant. Il est en effet inadmissible que des pratiques qui se veulent favorables au patient, comme son implication dans la prise de décision, puissent générer chez lui de la détresse psychologique, comme l'ont exprimé les participantes à notre étude. Lorsqu'une décision peut entrainer des événements aussi dramatiques que la mort, on se doit d'investir davantage de ressources à l'encadrement des femmes et de leurs proches, que ce soit pour le transfert d'information, la définition des besoins personnels ou la gestion des effets psychologiques pouvant apparaitre pendant le processus.

En somme, malgré des efforts considérables réalisés de part et d'autre de cette dyade patient-médecin pour rompre avec le modèle paternaliste et les rapports inégalitaires, il appert qu'un certain nombre de problématiques subsistent, lesquelles devront faire l'objet de recherches complémentaires auprès des femmes et des professionnels de la santé afin de trouver des solutions adaptées 
et d'en arriver à actualiser le modèle de prise de décision partagée. Le principal enjeu de ce modèle consiste donc principalement à ramener au centre de la dyade patient-médecin, dans un esprit de partenariat, le pouvoir de décision que l'on avait dirigé vers les femmes.

\section{Remerciements}

Les auteurs tiennent à remercier le Réseau de formation en recherche périnatale du Québec - IRSC (QTNPR) pour leur soutien financier pendant la rédaction. Cet organisme n'a joué aucun rôle dans la conception de l'étude, la collecte de données, l'analyse des données, l'interprétation des données, ou la rédaction du rapport.

\section{Note}

1 CHU Sainte-Justine, Département Obstétrique et Gynécologie, Université de Montréal, 3175 chemin de la Côte Ste-Catherine, Montréal (Québec) H3T 1C5, 1 (514) 345-4931 \#7574, Fax: 1 (514) 345-4668. Courriel: dugas11@hotmail.com

\section{Bibliographie}

AMERICAN CONGRESS OF OBSTETRICIANS AND GYNECOLOGISTS (2010). Ob Gyns Issue Less Restrictive VBAC Guidelines, réf. de janvier 2012, ACOG practice bulletin, 21 juillet 2010, http://www.acog.org/About_ACOG/News_Room/News_Releases/2010/ Ob_Gyns_Issue_Less_Restrictive_VBAC_Guidelines

BEKKER, Hilary L., Jenny HEWISON et Jim G. THORNTON (2004). «Applying decision analysis to facilitate informed decision making about prenatal diagnosis for Down syndrome: a randomised controlled trial ", Prenatal Diagnosis, Vol. 24, № 4, p. 265-275.

CAUGHEY, Aaron B. (2010). "Vaginal Birth After Cesarean Delivery ", réf. août 2011, http:// emedicine.medscape.com/article/272187-overview

CHAILLET Nils, et collab. (2007). "Identifying barriers and facilitators towards implementing guidelines to reduce caesarean section rates in Quebec ", Bulletin of the World Health Organization, Vol. 85, No10, p.791-797.

CHAILLET Nils, et collab. (2006). "Evidence-based strategies for implementing guidelines in obstetrics: A systematic review », Obstetrics \& Gynecology,Vol. 108, No 5, p. 1234-1245.

DELUCIA-WAACK Janice L., et collab. (dirs.) (2004). Handbook of group counseling and psychotherapy, Thousand Oaks, CA, Sage Publications, 744 p.

DEUTCHMAN Mark, et Richard G. ROBERTS (2003). «VBAC: Protecting Patients, Defending Doctors ", Amercan Family Physicians, March 1, 2003. 
DODD, Jodie M., et collab. (2007). «Birth after caesarean study - planned vaginal birth or planned elective repeat caesarean for women at term with a single previous caesarean birth: protocol for a patient preference study and randomised trial », BMC Pregnancy \& Childbirth, Vol 7, No17.

DUGAS, Marylène (2011). "Donner aux populations vulnérables de l'Afrique subsaharienne un accès aux soins obstétricaux : Stratégie avancée de consultations prénatales », Médecine tropicale, Vol. 71, No 6, p. 526-528.

DUGAS, Marylène, et Janice E. GRAHAM (2011). « Is consent for research genuinely informed? Using decision aid tools to obtain informed consent in the global South ", Journal of Global Ethics, Vol. 7, № 3, p. 349-359.

FRASER, William, et collab. (1997). « Randomized controlled trial of a prenatal vaginal birth after cesarean section education and support program. Childbirth Alternatives Post-Cesarean Study Group », American Journal of Obstetric and Gynecology, Vol.176, № 2, p. 419-425.

FROST, Jarvist M., et collab. (2009) «Women's views on the use of decision aids for decision delivery

following a previous caesarean section: qualitative interview study ", BJOG: An International Journal of Obstetrics \& Gynaecology,Vol. 116, № 7, p. 896-905.

GLAZIER, Richard, et collab. (1997). «Written patient information about triple-marker screening: A randomized controlled trial ", Obstetrics and Gynecology, Vol. 90, N 5, p.769-774.

GLAZIER, Richard, et collab. (2004). «Stress, social support, and emotional distress in a community sample of pregnant women ", Journal of Psychosomatic Obstetrics and Gynecology, Vol. 25, № 3-4, p. $247-255$

GOLDMAN, Gail, et collab. (1990). "Effects of patient, physician and hospital characteristics on the likelihood of vaginal birth after previous caesarean section in Quebec ", Canadian Medical Association Journal, Vol.143, N¹0, p.1017-1024.

HANNAH, Walter J., et collab. (1986). "Indications for cesarean section: final statement of the panel of the National Consensus Conference on Aspects of Cesarean Birth ", Canadian Medical Association Journal,Vol.134, No12, p.1348-1352.

HUNTER, A.G.W., et collab. (2005). " A randomized trial comparing alternative approaches to prenatal diagnosis counseling in advanced maternal age patients ", Clinical Genetics, Vol. 67, N ${ }^{\circ}$ 4, p. 303-313.

IGLESIAS, Stuart., Robert BURN et L. Duncan SAUNDERS (1991). "Reducing the cesarean section rate in a rural community hospital ", Canadian Medical Association Journal, Vol. 145, N ${ }^{\circ}$ 11, p.1459-1464.

KENTON, Kimberly, et collab. (2005). " Repeat cesarean section and primary elective caesarean section: Recently trained obstetrician-gynaecologist practice patterns and opinions ", American Journal of Obstetrics and Gynecology, Vol.192, № 6, p.1872-1876.

MARTEAU,Theresa M., et Hilary BEKKER (1992). «The development of a six-item short-form of the state scale of the Spielberger state-trait anxiety inventory (STAI) ", British Journal of Clinical Psychololgy, Vol. 31, № 3, p. 301-306.

MONTGOMERY,Alan A., et collab. (2007). «Two decision aids for mode of delivery among women with previous caesarean section: randomised controlled trial », BMJ, No 334, p.1305. 
NASSAR, Natasha, et collab. (2007). « On behalf of the Decision Aid for Breech Presentation Trial Collaborators. Evaluation of a decision aid for women with breech presentation at term: a randomised controlled trial ", BJOG: An International Journal of Obstetrics \& Gynaecology,Vol.114, $\mathrm{N}^{\circ} 3$, p.325-333.

O'CONNOR, Annette M., et M.J. JACOBSEN (2000). «Conflit décisionnel: évaluation et aide apportée aux clients confrontés à des décisions concernant leur santé ", réf. du 27 avril 2012, http://decisionaid.ohri.ca/francais/docs/Conflit_Decisionnel.pdf

O'CONNOR, Annette M, et collab. (2003). "Decision aids for people facing health treatment or screening decisions ", Cochrane Database of Systematic Revew, Vol.2, CD004131.

RICCIOTTI, Hope A., Katherine T.H. CHEN et Benjamin P. SACHS (1995). "The Role of Obstetrical Medical Technology in Preventing Low Birth Weight ", The Future of Children, Princeton-Brookings, Vol. 5, No. 1, p. 71-86.

ROBERTS, Richard G., et collab. (1997). «Trial of labor or repeated cesarean section. The woman's choice ", Archives of Family Medicine,Vol. 6, № 2, p.120-125.

SOCIÉTÉ DES OBSTÉTRICIENS ET GYNÉCOLOGUES DU CANADA (2005). «Directive clinique sur l'accouchement vaginal chez les patientes ayant déjà subi une césarienne ", Directives cliniques de la société, $\mathrm{N}^{\circ} 155$.

STEWART,A, et collab. (2003). "Assessment of the effect upon maternal knowledge of an information leaflet about pain relief in labor ", Anaesthesia,Vol. 58, № 10, p. 1003-1022.

THOMSON, Patricia, et collab. (2006). «A computerised guidance tree (decision aid) for hypertension, based on decision analysis: Development and preliminary evaluation ", European Journal of Cardiovascular Nursing, Vol. 5, No 2, p.146-149.

TWISSELMANN, Wiebke (2006). "Shared Decision Making, Décision partagée - pour une meilleure prise en charge? ", Primary Care, Vol. 6, No 39.

WONG, S, et collab. (2006). "Randomized controlled trial of a decision aid leaflet to facilitate women's choice between pregnancy termination methods ", BJOG: An International Journal of Obstetrics \& Gynaecology, Vol. 113, Nº, p. 688-694. 